\title{
Management of distal tibial intra-articular fractures with circular external fixation
}

\author{
G. Lovisetti - M. A. Agus - F. Pace • \\ D. Capitani $\cdot$ F. Sala
}

Received: 14 February 2008/ Accepted: 16 February 2009/Published online: 19 March 2009

(c) Springer-Verlag 2009

\begin{abstract}
The treatment of tibial plafond fractures requires careful management of the soft tissue envelope, reconstruction of the articular surface and stable fixation with minimal additional damage. Thirty cases of AO type $43 \mathrm{C}$ tibial fractures were treated by transosseous osteosynthesis (Ilizarov technique). The external fixator constructs used were Ilizarov (Transosseous osteosynthesis: theoretical and clinical aspects of the regeneration and growth of tissue, Springer, Berlin, 1992) and Sheffield (Classification AO des fractures, Springer, Berlin, 1987) circular fixator systems. All tibial plafond fractures healed. Using radiological criteria for assessment of reduction of the articular fragments and the clinical scoring system described by Teeny and Wiss, there were excellent and good restoration of articular structure in 27 cases and good clinical results in 14 . This treatment method compares well with previous published series and is to be recommended for this group of difficult fractures.
\end{abstract}

Keywords Distal tibial fractures - External fixation . Ilizarov $\cdot$ Minimally invasive

\author{
G. Lovisetti \\ Civil Hospital, Meneggio (Co), Milan, Italy \\ M. A. Agus - F. Pace \\ Milan University, Milan, Italy \\ D. Capitani \\ Niguarda Hospital, Milan, Italy \\ F. Sala $(\bowtie)$ \\ Nigurda Hospital, Milan, Italy \\ e-mail: sala.francesco@gmail.com
}

\section{Introduction}

The thin soft tissue envelope that surrounds the distal tibia makes treatment of these fractures difficult. These fractures are often referred to as "pilon" fractures [1] or "plafond" fractures" [2] if the articular surface of the tibia is involved; in such cases an anatomic realignment of the involved articular fracture in conjunction with a stable fixation is crucial [3-7]. The main mechanisms of injury for pilon fractures are two: (1) low-energy types, secondary to rotational forces (sporting accidents) and (2) highenergy types from axial loading of the distal articular surface against the talus causing multifragmental implosion of cartilage and bone (motor vehicle accidents, falls, work accidents). The plafond area in which the fracture develops depends on the position of the foot at the moment of trauma $[5,8]$. There may be associated fractures of the talus, calcaneus, pelvis or spine. In 1953 Lauge Hansen described a four stage classification based on the progression of a pronation dorsiflexion mechanism [9]. Rüedi and Allgöwer [5] proposed a three stage classification in which the degree of comminution and articular incongruity play a fundamental role [3]. This classification has been widely used and modified by Ovadia and Beals in a five-stage classification in 1986 [10]. We consider the classification proposed by Müller and Nazarian in 1987 to be the most complete and detailed [11].

Fractures of the distal tibia have been treated in the past using various modalities. Rüedi and Allgöwer presented good results with open reduction and stable internal fixation using plates and screws [4]. With the increasing incidence of high-energy injuries, however, a rise in complications when using such treatment has been observed including soft tissue dehiscence, infection, osteomyelitis, delayed union or nonunions [12-14]. Minimally 
invasive techniques for reduction of the articular fragments combined with stable fixation through an external device have been employed in more recent years [15-23]. Circular frames with tension wires, like the classic Ilizarov fixator, provide better stabilisation especially in comminuted lesions and control the fracture in all three planes of the reduction [24-31]. The use of olive wires enable multidirectional forces to be applied for reducing syndesmotic separations, tibial malleolar fragments and can even provide horizontal compression to a spiral fracture pattern. Inclusion of the foot in the external fixator and joint spanning through hinges are also possible with these circular external fixator designs. The purpose of this study is to present the results of circular external fixation combined with internal fixation in these injuries.

\section{Materials and methods}

Thirty articular fractures of distal tibia were treated in the period from 1998 to 2007 at the Sant'Anna and Cà Granda Niguarda Orthopaedic Departements of Como and Milan. Twenty cases had a classic Ilizarov ring fixator (Plusteck srl, Milano Italy). The remaining ten cases were treated with a Sheffield (SRF) ring fixator (Orthofix srl, Verona Italy). There were 23 males and 7 females, with a mean age of 53 years (range: 26-72 years). All fractures had 2 standard x-rays of the entire leg and standard and oblique projections of the ankle.

Two patients were $2 \mathrm{HCV}$ positive, 4 were HIV positive ( 1 with AIDS), 2 were known to abuse alcohol and there were 4 heavy smokers.

Using the Müller-Nazarian classification, there were twenty-nine $43 \mathrm{C}$ patterns (C1: 11, C2: 9, C3: 10). Eight fractures were open fractures of the Gustilo and Anderson type I (4) and type II (4). Internal osteosynthesis was used in 7 cases ( 3 plates and 2 Rush pins for the lateral malleolus, 3 cannulated screws for the posterior malleolus, 1 medial malleolar screw, 8 cannulated screws for remaining fragments of the plafond). In none of the cases was bone graft employed. None of the patients required plastic surgery. Clinical follow up was performed at a median of 16.9 months after frame removal (range: 6-24 months).

\section{Operative technique}

Surgery is usually performed with the patient under general anaesthesia. The patient lies supine on the operative table with trans-calcaneal traction. The amount of reduction achieved by ligamentotaxis is checked using by intensification (Fig. 1a, b). If internal fixation is required for the
Fig. 1 a Fracture 43C 3 . b The amount of reduction achieved by ligamentotaxis
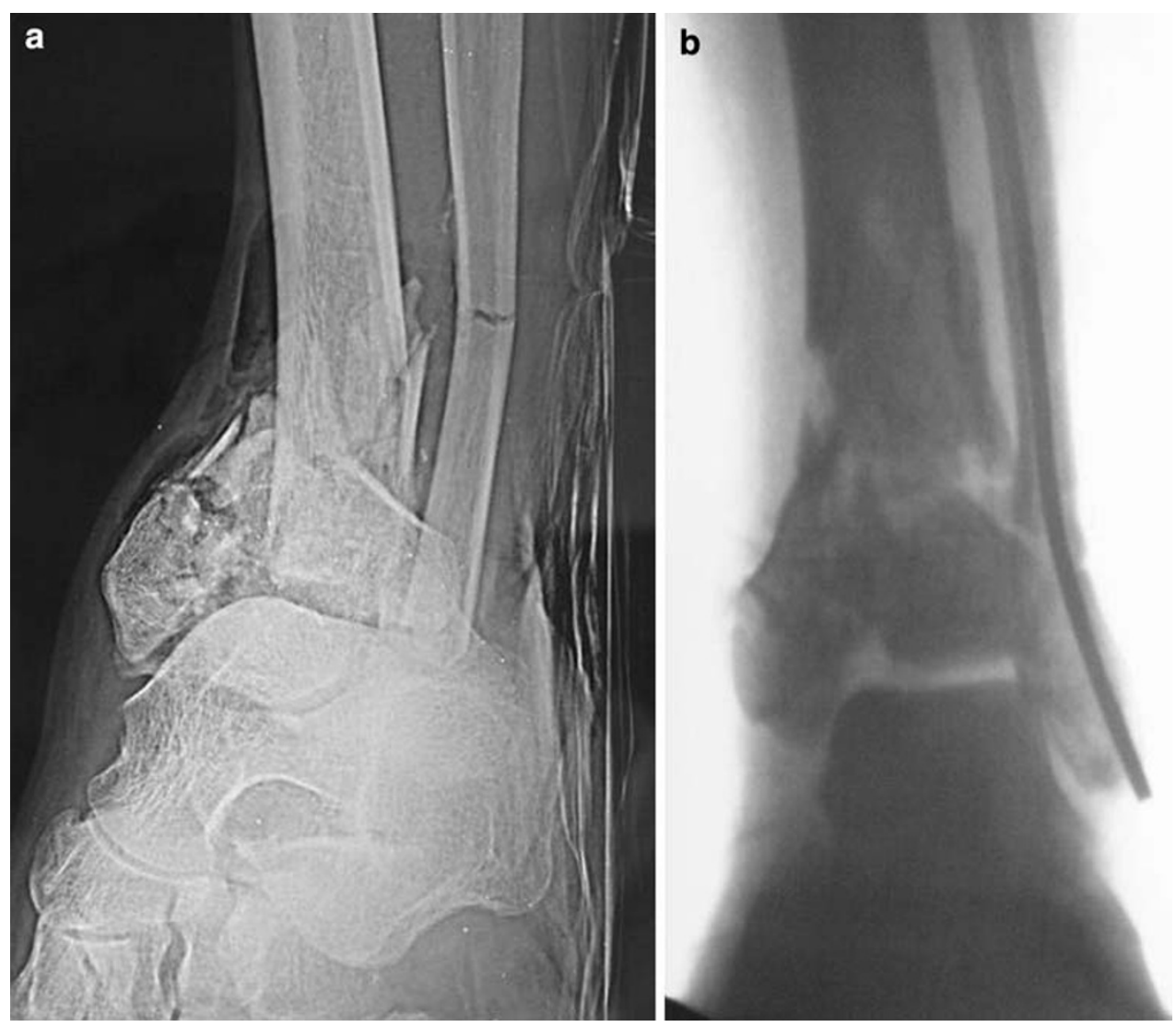


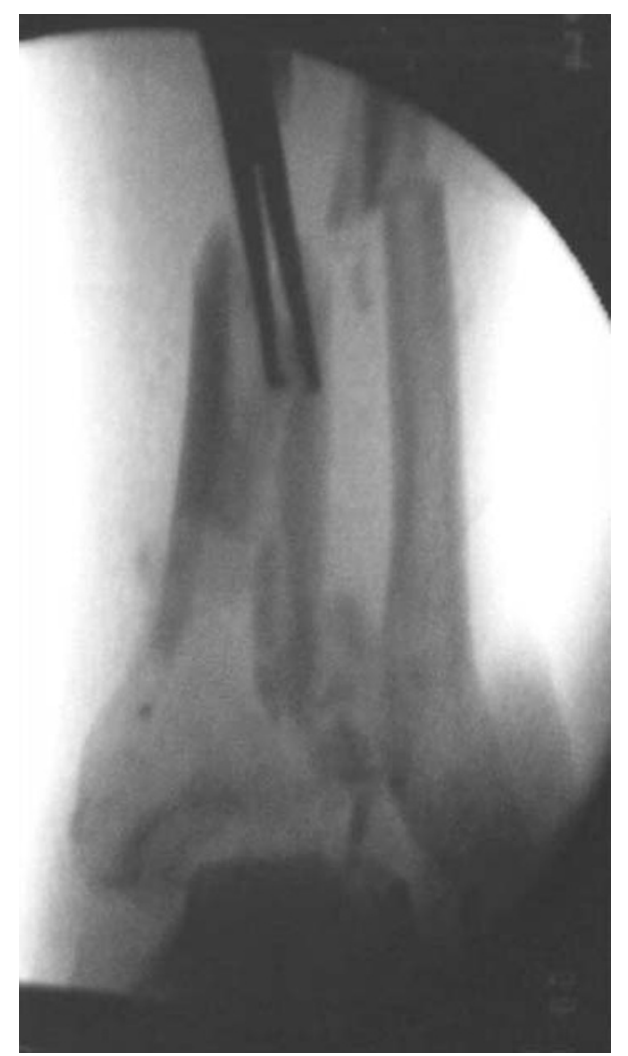

Fig. 2 The fragments can be replaced in correct position, under fluoroscopic check, with the aid of a clamp and a small incision over the fracture

lateral malleolus, this procedure is performed first to restore fibular length. Great care must be taken in accurate reduction of the "Tillaux" fragment; reduction can be obtained with minimally invasive manoeuvres performed with a smooth $3 \mathrm{~mm}$ wire used as a joystick and stabilised with $1.8-2.0 \mathrm{~mm}$ wire or with a cannulated screw. When distal migration of fragments is noted, these fragments can be replaced in the correct position (using fluoroscopic check) with the aid of a clamp inserted proximally through a minimal skin incision (Fig. 2). An olive wire can also be introduced through the tibial malleolus, first to reduce the fracture (usually tilted in varus and anterior) and then to compress the fracture site (Fig. 3). Alternatively, internal osteosynthesis of the medial malleolus, if feasible, is performed. Once reduction of the anterior fragments is accomplished, displacement of the posterior malleolus is achieved with dorsiflexion of the foot. The reduction is maintained with a guide wire for subsequent insertion of a cannulated screw. After restoration of articular congruity, a $1.8 \mathrm{~mm}$ Ilizarov wire ( $2.0 \mathrm{~mm}$ if $\mathrm{SRF}$ ) is passed parallel to the articular surface in the AP view on X-ray, approximately $2 \mathrm{~cm}$ from the joint line in the tibial epiphysis. The circular frame is then assembled on this wire and the injured limb. The use of carbon fibre rings facilitates radiographic assessments. The alignment is continually

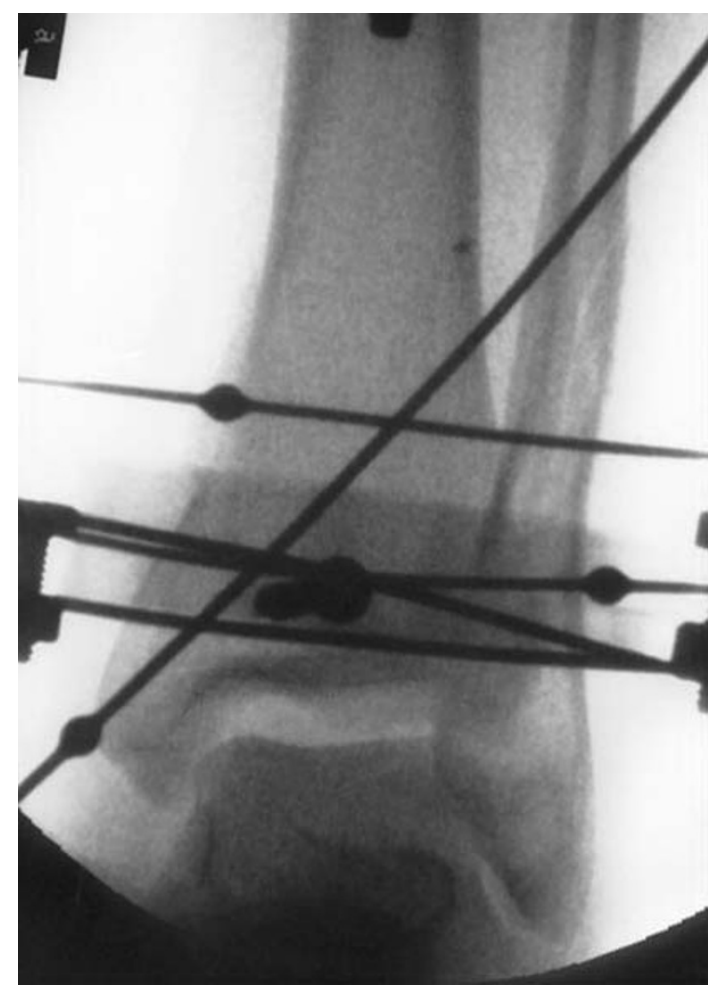

Fig. 3 Printout of intraoperative image intensification demonstrating the olive wires used to reduce the peroneo-tibial diasthasis, the spiral metaphyseal fracture and the tibial malleolus

checked by image intensification in both planes. Additional smooth or olive wires are inserted in safe corridors to improve the alignment and increase stability. On the distal tibial ring at least three wires are required. On the proximal block, half pins are inserted in combination with wires. The wires are fixed to the rings of the fixator and tensioned. When necessary the foot is incorporated by wires and screws inserted in the calcaneus and fixed to a half ring connected to the tibial external fixator. The foot is fixed in neutral position to avoid supination and equines position (Fig. 3). Following placement and tensioning of these main wires, the remaining displaced fragments can be gently reduced by inserting additional wires in a manner that allows reduction forces to be applied through the displaced fragment. In multi-fragmental fractures great care must be taken to avoid reducing a fragment with a wire that 'locks' another fragment in a nonreduced position. When olive wires are used, usually in an opposite (crossing) configuration, variable degrees of interfragmentary compression can be achieved. This is of particular benefit in reducing tibio-fibula diastasis and in putting transverse compressive forces on a spiral metaphyseal fracture (Fig. 4). When the distal ring is adjacent at the level of the ankle joint, impingement with the dorsal surface of the foot during ankle motion must be avoided. At the end of the procedure the degree of the ligamentotaxis must be greatly reduced to 


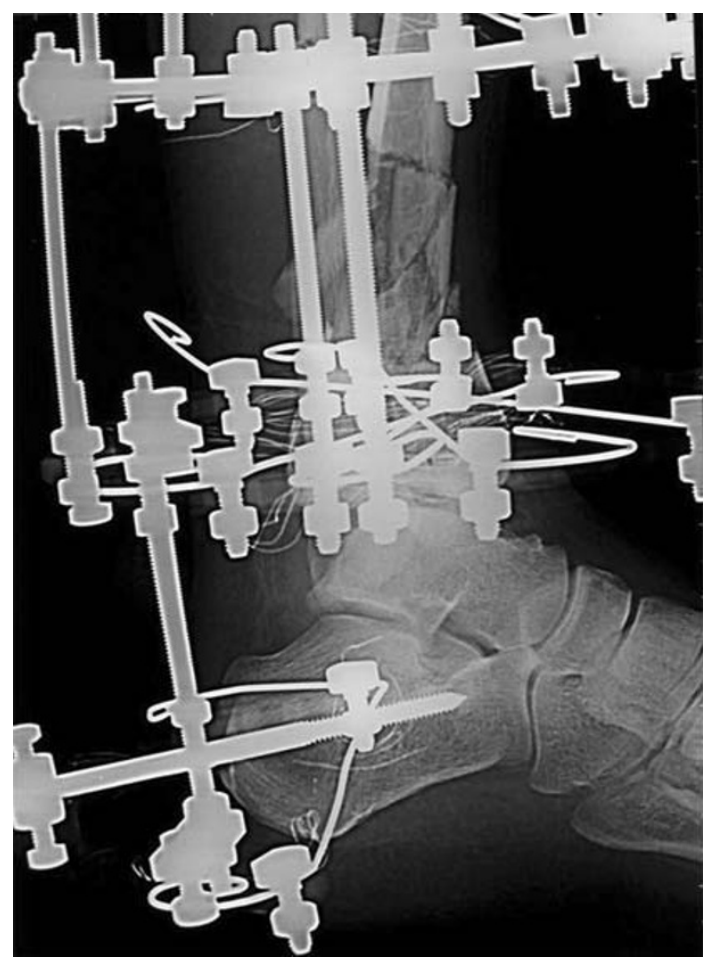

Fig. 4 A half-pin inserted axially in the calcaneus fixed to a half ring connected to the hardware was used to incorporate the foot

avoid neurodystrophic disorders of the foot. We normally use 4 rings for the Ilizarov fixator and 2 or 3 rings for SRF device.

\section{Postoperative care}

The foot ring, if used, can be removed after an average of 40 days. Physiotherapy is started to avoid the development of an equinus posture of the foot. Partial weight bearing is allowed after 45 days (60 days in highly comminuted articular lesions).

The patients had care of the pin site according to the Russian Ilizarov Scientific Centre for Restorative Traumatology and Orthopaedics [32].

\section{Results}

The accuracy of reduction of the articular fragments was assessed according to criteria described by Ovadia and

Table 1 Quality of reduction

\begin{tabular}{lll}
\hline Anatomic & 8 points & 5 cases \\
Good & $9-11$ points & 23 cases \\
Fair & $12-15$ points & 2 cases \\
Poor & $>15$ points & - \\
\hline
\end{tabular}

Table 2 Clinical rating

\begin{tabular}{lll}
\hline Excellent & $>92$ & 6 cases \\
Good & $87-92$ & 9 cases \\
Fair & $65-86$ & 2 cases \\
Poor & $<65$ & 2 cases \\
\hline
\end{tabular}

Beals and clinical outcome according to Teeny and Wiss $[10,12]$. Reduction was judged anatomic (8 points), good (9-11 points), fair (12-15 points) or poor (more than 15 points). Accuracy of reduction scored a mean of 9.45 points (range 8-13); it was judged anatomic in 5 cases, good in 23 and fair in 2 patients (Table 1). Soft tissue healing occurred without need for plastic surgery in all cases. The external fixator was removed after a mean of 21.4 weeks (range 10-41). Union occurred in all cases.

There were no diagnoses of deep venous thrombosis but superficial pin tract infections were present in 5 cases and resolved in 4 using local antiseptic and an oral antibiotic regimen. Infection at 3 pin sites from the distal tibial ring was encountered in one case with pin loosening evident after 4 months. We replaced the half screws with wires.

Clinical results revealed a mean score of 81.5 points (range 58-100); it was judged excellent in 6 patients, good in 9 , fair in 13 and poor in 2 cases (Table 2). Of these, the first required an arthrodesis of the ankle. This patient, despite having a quality of reduction scored at 9 (good), developed a stiff ankle with equinus of the foot from interruption of physiotherapy consequent to admission to hospital following a myocardial infarction. The second poor result was a 34-year-old man with $43 \mathrm{C} 3$ and diaphyseal fractures in the same leg treated with Ilizarov apparatus. He had soft tissue injuries with Gustillo IIIA tibial exposition in the middle of the leg. There was union between the epiphyseal and metaphyseal segments of the fracture but a delayed union at the level of the tibial diaphyseal fragments. Successful conclusion to the treatment was accomplished by revision to bifocal Ilizarov technique performed four months later the first surgical treatment. Other complications included a varus deformity of the tibial callus of $10^{\circ}$ after frame removal at 90 days in a $43 \mathrm{C} 3$ fracture in a male aged 55 years who smoked heavily. The clinical result was however found to be excellent. A $5^{\circ}$ valgus deformity also occurred after Ilizarov apparatus removal in a 31 years woman with a $43 \mathrm{C} 2$ fracture. A short leg cast was applied for 25 days and the final clinical result judged good.

\section{Discussion}

Open reduction and internal fixation (ORIF) has led to variable results in high-energy distal tibia fractures, especially in comminuted articular or open types. An open 
reduction and stable fixation of these fractures was proposed by Rüedi and Allgöwer in 1969; they treated 84 intra-articular fractures which were not classified radiologically and 60 were of a low-energy mechanism (sport injury, e.g. ski accidents, etc.). These authors described excellent or good results in $74 \%$ of cases and an infection rate of 5\% and wound healing problems in $12 \%$ [3]. They subsequently published a second group of 75 patients in 1979 of which $47 \%$ were grade III and $34 \%$ of high energy type [5]. A favourable observer-determined outcome was reported in $69.4 \%$ of cases and without complications of deep infection. They were the first to describe a systematic approach to the "pilon problem" and added a useful method of classification and emphasised the importance of anatomic articular restoration. Subsequent studies failed to reproduce these favourable outcomes and other authors have criticised the overall approach. In complex lesions, an extensive surgical approach which facilitates insertion of large plates may further compromise the viability of bone and the damages soft tissues. Consequently, an unacceptable percentage of wound dehiscence problems and deep bone infection have been reported to occur [12, 33, 34]. Teeny and Wiss in 1993 found unsatisfactory results of ORIF in type III Rüedi fractures in a group of 58 patients [12]. There was a $37 \%$ incidence of wound dehiscence, infection in $37 \%$, nonunion in $27 \%$ and poor consolidation in $23 \%$. In contrast, they observed no infection but wound problems in $17 \%$, nonunion in $7 \%$ and delayed consolidation in $3 \%$ of fractures of types I and II. Ovadia and Beals presented a large report of 142 fractures (45\% high energy, $10 \%$ open), and expanded the Rüedi classification to a 5-type system (types I, II and III corresponding to types I and II, and types IV and V to grade III). These fractures were treated with ORIF and other techniques. In type I, a favourable outcome was reported in $100 \%$ of cases whereas in type $\mathrm{V}$ only $22 \%$. Poor results of ORIF were also reported by Wyrsch et al. [35] in a randomised study in which the ORIF method was compared to external fixation for two groups of 19 and 20 patients, respectively. In the ORIF group, an incidence of $28 \%$ of deep infection, $33 \%$ wound dehiscence and $16 \%$ amputation rate were reported. In the external fixation group the infection rate was 5\% (a septic arthritis of the ankle was observed) and no amputations. In the ORIF group patients underwent surgery 3-5 days after trauma, a period considered risky by the majority of the authors, while in the external fixation group surgery was performed immediately or after a mean of 7 days. This difference may confound the results described as the timing of open surgery on high-energy fractures of the distal tibia is critical [35]. Mast et al. [36] and Trumble et al. [19] chose to perform surgery in the first 8-12 h whereas a two-step method was proposed by Patterson and Cole [37]; the lateral malleolus is stabilised first with internal fixation and the tibia by spanning external fixation, followed by definitive reconstruction and stabilisation of the fracture 10-14 days later [19, 36, 37]. External fixation techniques preserve soft tissues and the periosteum and yet provide stable reduction. The risk of non union and malunion in presence of instability is higher. In a report by Dillin and Slabaugh, there was 55\% infection and $36 \%$ wound dehiscence rate with unstable internal fixation [38]. Bonar and Marsh [39, 40] treated a group of 49 patients by with trans-articular external fixation and used limited surgical exposures to accomplish a small degree of internal osteosynthesis[39, 40]. They described an anatomic reduction in none, a good reduction in $69 \%$, fair in $20 \%$ and poor in $11 \%$. Barbieri et al. also treated 37 fractures in 36 patients with external fixation and minimal internal osteosynthesis [15]. Internal osteosynthesis of tibia was performed in $56 \%$ of cases and of fibula in $80.7 \%$; an anatomic reduction was obtained in all but one case. These authors have employed internal osteosynthesis to a greater percentage of cases than in our series. Soft tissue complications and deep infections are not frequent when external fixation is combined with minimally invasive surgery: Wyrsch had infections in 5\%, Tornetta in 1 out of 17 cases, Barbieri in 3 out of 37 cases. In this series, there were no cases of pseudoarthrosis or deep infection. The mean union time was 21.4 weeks. We attribute the $100 \%$ union rate to meticulous preservation of soft tissues in the fracture zone, made possible by the strategy of treatment. Despite the quality of reduction achieved and lack of complications observed, the clinical outcomes in our series have been less favourable than others; there were excellent and good results reported in 14 out of 29 cases and two cases with poor outcomes. This may highlight the heterogeneity within this fracture group and indicate that outcomes may not always parallel the quality of reduction achieved as assessed by radiograph. In conclusion, the authors deem that treatment of pilon fractures by circular external fixation (in accordance with Ilizarov principles) with or without minimal internal fixation allows for less soft tissue dissection and represents a reliable method for achieving stabilization and healing of distal tibial intra-articular fractures with a minimum of soft tissue complications.

\section{References}

1. Destot E (1911) Traumatismes du pied et rayons x malleoles, astragale, calcaneum, avant-pied. Paris, Masson, pp 1-10

2. Bonin JG (1950) Injuries to the ankle. William Heinemann, London, pp 248-260

3. Ruedi TP, Allgoewer M (1969) Fractures of the lower end of the tibia into the ankle joint. Injury 1:92

4. Ruedi TP, Allgoewer M (1973) Fractures of the lower end of the tibia into the ankle joint: results 9 years after open reduction and internal fixation. Injury 5:130 
5. Ruedi TP, Allgoewer M (1979) The operative treatment of intraarticular fractures of the lower end of the tibia. Clin Orthop 138:105

6. Kellam JF, Waddel JP (1979) Fractures of the distal metaphysis with intra-articular extension. The distal tibia explosion fracture. J Trauma 19:593

7. Vives P, Hourlier H, De Lestang M et al (1984) Eighty-four fractures of the lower end of the tibia in adults. Attempt at a classification. Rev Chir Orthop Reparatrice Appar Mot 70:129-139

8. Helfet D, Koval K, Pappas J, Sanders WR, DiPasquale T (1994) Intra-articular pilon fracture of the tibia. Clin Orthop 298: 221

9. Lauge-Hansen N (1953) Fractures of the ankle V: pronation dorsiflexion fracture. Arch Surg 67:813

10. Ovadia DN, Beals RK (1986) Fractures of the tibial plafond. J Bone Joint Surg. 68A:543

11. Mueller ME, Nazarian S, Koch P (1987) Classification AO des fractures. Springer Verlag, Berlin, pp 170-179

12. Teeny SM, Wiss DA (1993) Open reduction and internal fixation of tibial plafond fractures: variables contributing to poor results and complications. Clin Orthop 292:108

13. Rommens PM, Claes P, DeBoodt P, Stappaerts KH, Broos PL (1994) Therapeutic procedure and long term results in tibial pilon fracture in relation to primary soft tissue damage. Unfallchirurgie 97:39-46

14. Pollak AN, McCarthy ML, Bess RS, Agel J, Swiontkowski MF (2003) Outcomes after treatment of high-energy tibial plafond fractures. J Bone Joint Surg Am 85:1893-1900

15. Barbieri R, Schenk R, Koval K, Aurori K, Aurori B (1996) Hybrid external fixation in the treatment of tibial plafond fractures. Clin Orthop 332:16

16. Bone LB (1987) Fractures of the tibial plafond. The pilon fracture. Orthop Clin North Am 18:95

17. Bone L, Stegemann P, McNamara K, Seibel R (1993) External Fixation of severely comminuted open tibial pilon fractures. Clin Orthop 292:101

18. Tornetta P, Weinwer L, Bergman M et al (1993) Pilon fractures: treatment with combined internal and external fixation. J Orthop Trauma 7:489-496

19. Trumble TE, Benirschke SK, Vedder NB (1992) Use of radial forearm flaps to treat complications of closed pilon fractures. J Orthop Trauma 6:358

20. Ayeni JP (1988) Pilon fractures of the tibia: a study based on 19 cases. Injury 19:109-114

21. Nast-Kolb D, Betz A, Rodel C, Schweiberer L (1993) Minimal osteosynthesis of tibial pilon fracture. Unfallchirurgie 96:517-523

22. Dickson KF, Montgomery S, Field J (2001) High energy plafond fractures treated by a spanning external fixator initially and followed by a second stage open reduction internal fixation of the articular surface-preliminary report. Injury 32(Suppl. 4):SD92SD98

23. Blauth M, Bastian L, Krettek C, Knop C, Evans S (2001) Surgical options for the treatment of severe tibial pilon fractures: a study of three techniques. J Orthop Trauma 15(3):153-160
24. Catagni MA (1991) Fractures of the leg. In: Bianchi Maiocchi A, Aronson J (eds) Operative principles of Ilizarov. Medicalplast. p 91

25. Catagni MA, Malzev V, Kirienko A (1994)Treatment of distal articular fractures of tibia/fibula (tibial plafond fractures) in Advances in Ilizarov apparatus assembly Editor Bianchi Maiocchi A. Medicalplast p 55

26. Cattaneo R (1985) La technique d'Ilizarov dans les fractures de jambe. Actualité sur les fractures de jambe. XIIIe Journées de chirurgie orthopédique et traumatologique del l'Hopital Bichat. p129

27. Ilizarov GA (1992) Transosseous Osteosynthesis: theoretical and clinical aspects of the regeneration and growth of tissue. Springer, Berlin

28. Gaudinez R., Mallik AR, Szporn M (1996) Hybrid external fixation in tibial plafond fractures. Clin Orthop Relat Res 329:223-232

29. Saleh M, Shanahan MDG, Fern ED (1993) Intra-articular fractures of the distal tibia: management by limited internal fixation and articulated distraction. Injury 24(1):37-40

30. Court-Brown C, Walker C, Garg A et al (1999) Half ring external fixation in the management of tibial plafond fractures. J Orthop Trauma 13(3):200-206

31. El-Shazly M, Dalby-Ball J, Burton M, Saleh M (2001) The use of trans-articular external fixation for management of distal tibial intra-articular factures. Injury 32:99-106

32. Davies R, Holt N, Nayagam S (2005) The care of pin sites with external fixation. J Bone Joint Surg 87-B:716-719

33. Bourne RG, Rorabeck CH, MacNab J (1983) Intra-articular fractures of the distal tibia: the pilon fracture. J Trauma 23(7):591-596

34. Borner M (1982) Classification, treatment and results of fractures of the tibial pilon. Unfallchirurgie 8:230-235

35. Wyrsch B, McFerran MA, McAndrews M, Limbird TJ, Harper MC et al (1996) Operative treatment of fractures of the tibial plafond. A randomized prospective study. J Bone Joint Surg 78A:1646-1657

36. Mast JW, Spiegel PG, Pappas JN (1988) Fractures of the tibial pilon. Clin Orthop 230:68

37. Patterson M, Cole JD (1999) Two-staged delayed open reduction and internal fixation of severe pilon fractures. J Orthop Trauma 13:85-91

38. Dillin L, Slabaugh P (1986) Delayed wound healing, infection and non-union following open reduction and internal fixation of tibial plafond fractures. J Trauma 26:1116

39. Bonar SK, Marsh GL (1993) Unilateral external fixation for severe pilon fractures. Foot Ankle Int 14:57

40. Marsh JL, Weigel DP, Dirschl DR (2003) Tibial plafond fractures: how do these ankles function over time? J Bone Joint Surg 85A:287-295 